\title{
Pacific
}

Journal of

Mathematics

\section{LENGTH OF JULIA CURVES}

\author{
DAVID H. HAMILTON
}

Volume 169 No. 1 


\title{
LENGTH OF JULIA CURVES
}

\author{
David H. Hamilton
}

\begin{abstract}
Let the Fatou set of a meromorphic function $f$ have two components with Denjoy-Wolff points at which $f$ is not transcendental. Then the Julia set $J$ is a "circle/line" or is nowhere rectifiable. In particular if $f$ is rational and $J$ is a Jordan curve then it follows it follows (in the second case) that $\operatorname{dim}(J)>1$.
\end{abstract}

Let $F: \mathbb{C} \rightarrow \mathbb{C}$ be a meromorphic function. The Julia set $J=J(F)$ is the complement of the set of normality of the iterates $F^{n}$. Fatou (1920) proved that if $F$ is rational and if $J$ is a closed Jordan curve then either $J$ is a "circle/line" or it has a dense set of points where there is no tangent. Under various special assumptions on the behaviour of $F$ on $J$ one can say more about the case where $J$ is a closed Jordan curve not equal to a circle/line: Fatou claimed that if " $F$ is expanding on $J$ " then $J$ has Hausdorff 1-measure $\lambda(J)=\infty$. (This was proved by Brolin (1965).) Also if " $F$ is expanding on $J$ " Sullivan (1983) proved that the Hausdorff dimension satisfies $\operatorname{dim}(J)>1$. Sullivan does this by applying his construction of the conformally invariant measure to Brolin's result. The main result of this paper is that these results hold for arbitrary rational functions.

ThEOREM 1. Let $F: \mathbb{C} \rightarrow \mathbb{C}$ be a rational function. Suppose that the Julia set $J$ is a Jordan curve. Then $J$ is a circle/line or $\operatorname{dim}(J)>1$.

Remarks. (i) Actually Przytcki, Urbanski and Zdunik [14] had proved Theorem 1 for the "repellant case". Also Zdunik [17] proves that if $F$ is any polynomial with Julia set $J$ a Jordan curve then $\operatorname{dim}(J)>1$. We only prove $\lambda(J)=\infty$ and then make good use of Decker and Urbanski [5].

(ii) Note that we require $J$ to be a closed Jordan curve. Our result is considerably more general than for Jordan curves. We only require that $F$ has two foreward invariant (disjoint simply connected) 
domains $\Omega_{1}$ and $\Omega_{2}$. Then we prove that either $\mathbb{C}-\left(\Omega_{1} \cup \Omega_{2}\right)$ is a circle/line or $\lambda\left(\mathbb{C}-\left(\Omega_{1} \cup \Omega_{2}\right)\right)=\infty$. The case that $J$ is a Jordan arc has to be treated separately and follows from a general result we prove for "dendrites", i.e. when both $J$ and $\mathbb{C}-J$ are connected.

Our method depends primarily on the harmonic measure

$$
\omega_{j}=\omega_{j}\left(z_{j}, E, \Omega_{j}\right),
$$

i.e. the value of the function harmonic on $\Omega_{j}$ with boundary value 1 on $E$ and 0 on $\partial \Omega_{j}-E$ taken at some point $z_{j}$ inside $\Omega_{j}$. The actual points $z_{j}$ are unimportant so we henceforth refer only to the harmonic measures $\partial \omega_{j}$. Our basic result is:

THEOREM 2. Let $F$ be a rational function with two foreward invariant (disjoint) domains $\Omega_{1}$ and $\Omega_{2}$. Then either $\partial \Omega_{1}=\partial \Omega_{2}$ is a circle/line or

$$
d \omega_{1} \perp d \omega_{2}
$$

REMARKS. (i) This immediately implies (by classical results of Riesz, see [13]) that in the second case $J$ is nowhere rectifiable. However we can improve this considerably by using results of Bishop, Carleson, Garnett and Jones [3]. They characterise the disjoint domains for which the harmonic measures are mutually singular. Let $T$ be the set of points on $J$ where there is a tangent. If the mutual boundary is a closed Jordan curve $J$ singularity is equivalent to $\lambda(T)=0$. In the general case one only has to replace tangents by "mutual sectorial accessability". In any case there is no "tangent" at almost all points, considerably strengthening the old result of Fatou.

We prove Theorem 2 directly as a consequence of our rigidity theory for inner functions $f$, see [8], [9] and [10]. Now $f$ is inner if it is a bounded analytic function on the unit disk $\mathbb{D}$ with radial boundary values $f(z) \in \partial \mathbb{D}$ for $z \in \partial \mathbb{D}$ (a.e.). Shub and Sullivan [15] dealt essentially with the special case where $f$ is rational, i.e. a finite Blaschke product, and $f$ fixes a point inside the disk. They prove that if there is a (mutually) absolutely continuous map $\phi: \partial \mathbb{D} \rightarrow \partial \mathbb{D}$ so that $g=\phi \circ f \circ \phi^{-1}$ is also Blaschke (on $\partial \mathbb{D}$ ) then $\phi$ is Mobius (bilinear or the complex conjugate of a bilinear 
map). In the general case the real difficulty is dealing with functions with "Denjoy-Wolff" point on $\partial \mathbb{D}$, the generalization to inner functions being straight foreward. The general result is that inner $f$ have nontrivial conjugations by absolutely continuous $\phi$ to other inner functions if and only if $f$ is not ergodic on $\partial \mathbb{D}$. By using the techniques of this method we shall obtain the stronger result on the singularity of harmonic measures.

Before proceeding consider some examples. If the Julia set $J$ of a rational function $f$ is a circle/line then $f$ is essentially a Blaschke product. Examples where $J$ is not a circle/line may be obtained by quasiconformal conjugation of a Blaschke product to a rational function $F$. Now if the fixed point is not on the Jordan curve $J$ we have the expanding case. So this theory is new only for the case where we have a neutral attracting point, i.e. petals. Similar results are also seen in the usual family of suspects $z^{2}+c$. For $c$ close to 0 we have that $J$ is a closed Jordan curve surrounding a strongly attracting point. However for some values like $c=1 / 4$ although $J$ remains a Jordan curve the bounded attracting point is also on $J$. For other values of $c$ we obtain the so called "rabbit ears". In this case $f^{2}$ is invariant on two bounded domains $\Omega_{1}$ and $\Omega_{2}$ as well as one unbounded domain $\Omega_{3}$. Now Theorem 2 implies that the relevant harmonic measures are mutually singular. This is hardly surprising for $\Omega_{1}$ and $\Omega_{2}$ as they only touch at one boundary point. However for $\Omega_{1}$ and $\Omega_{3}$ this is nontrivial. (Now we do not necessarily have that $\partial \Omega_{j}$ have infinite 1-measure, only that if they do have finite 1-measure then they meet on a set of zero measure.)

Also Theorems 1 and 2 will also be seen to hold for a large class of meromorphic functions $F$. However we need to restrict ourselves to meromorphic functions which are ergodic with respect to the harmonic measure on the invariant domains.

Now $F$ is ergodic if there is no set $E \subset \partial \Omega_{j}$ with

$$
0<\omega_{j}(E)<1 \text { and } F^{-1}(E)=E .
$$

A sufficient condition for this is that the "Denjoy-Wolff" point for $F$ on a foreward invariant domain $\Omega$, i.e. the point $w$ in the closure of $\Omega$ so that for all $z$ in $\Omega F^{n}(z) \rightarrow w$, should be a finite point (so that $F$ is analytic at w). The function $F=\tan (z)$ is an example. However there are meromorphic functions, such as $F=2 z+\tan (z)$ 
(with "Denjoy-Wolff" point at $\infty$ ) which are not ergodic.

THEOREM 3. Let $F$ be a meromorphic function with two foreward invariant (disjoint) domains $\Omega_{1}$ and $\Omega_{2}$ upon which $F$ is ergodic with respect to the harmonic measures $d \omega_{j}$. Then either $\partial \Omega_{1}=\partial \Omega_{2}$ is a circle/line or the harmonic measures are singular.

REMARKS. (i) We also give an example of a meromorphic function $F$ with Julia set $J$ being a Jordan curve separating the plane into forward invariant unbounded domains with harmonic measures which are mutually absolutely continuous but $J$ is not a circle/line. Indeed the harmonic measures are absolutely continuous with respect to $d \lambda$ on $J$. However we do not know if such $J$ can be of locally finite lengh (although they are never smooth).

Finally we deal with the case where $J$ is a Jordan arc or even a "dendrite". The general case is for a simply connected foreward invariant domain $\Omega$. In many situations the points of $\partial \Omega$ are accessible from several different directions, i.e. to be more precise several prime ends correspond to the same boundary point. For example if $J$ is a Jordan arc then (except at the end points) two prime ends correspond to each point of $J$. At the opposite extreme is the "rabbit" where a countable number of prime ends cut off some of the boundary points. Although we cannot speak of a homeomorphism of the circle generated by this correspondence we can obtain a bijection. Thus as above we shall prove:

THEOREM 4. Let $F$ be a rational function so that the Julia set $J$ and its complement are connected. Then $J$ is a circular arc/line interval or $\lambda(J)=\infty$.

REMARKS. Actually one proves that the resulting bijection is trivial or singular. If $J$ is actually a Jordan arc then Theorem 4 also holds for meromorphic functions.

The author would like to thank the referee and acknowledges conversations with M. Yacobson and P.W. Jones.

2. Conformal Pullback to the disk. Let $F$ be meromorphic on $\mathbb{C}$ with Julia set $J$. We consider a foreward invariant simply connected component $\Omega$ of $\mathbb{C}-J$. Let $h$ be the conformal mapping of the unit disk $D$ onto $\Omega$. We have by Fatou's Theorem 


$$
\lim _{r \rightarrow 1} h(r z) \in \partial \Omega, z \in \partial \mathbb{D} \text { (a.e.). }
$$

Consequently we have:

LEMMA 1. The function

$$
f \equiv h^{-1} \circ F \circ h
$$

is a well defined inner function of $\mathbb{D}$ into $\mathbb{D}$.

Now $f$ is a well defined analytic map of the unit disk into itself. So we have only to check that $f$ is inner. If not there is a subset $E$ of the circle with $\lambda(E)>0$ and

$$
\lim _{r \rightarrow 1} f(r z) \in \mathbb{D}, x \in E \text { (a.e.). }
$$

Then the radial limits

$$
G \equiv h\left(E_{0}\right), \lambda\left(E-E_{0}\right)=0,
$$

is a subset of $\partial \Omega$ but $F(G) \subset \Omega$ ! This of course is impossible.

Notice that $f$ is bilinear if and only if $F$ is conformal on $\Omega$. Using the complete classification of dynamical behaviour this happens only if there is a bilinear mapping $T$ of the disk onto itself so that $f=$ $T \circ g \circ T^{-1}$ where

$$
g(z)=e^{i t} z
$$

where $t / \pi$ is irrational. Thus $f$ is ergodic, i.e. fixes no nontrivial set of positive length on $\partial \mathbb{D}$.

Now if $F$ fixes a point $w$ of $\Omega$ then by the principle of harmonic measure the measure $d \omega(w, z, \Omega)$ is $F$ invariant. A simple argument shows that $f$ is ergodic with respect to this finite measure.

Doering and Mané [6] considered the case for parabolic basins of rational functions and proved the corresponding $f$ is ergodic on the unit circle even though there is no invariant finite measure. Their proof only depends on the following sufficient condition due to Aaronson [1]:

LEMMA A. An inner function $f: \mathbb{D} \rightarrow \mathbb{D}$ is ergodic provided 


$$
\sum_{n=1}^{\infty}\left(1-\left|f^{n}(0)\right|\right)=\infty
$$

REMARK. Actually, see [7], this is equivalent to $f$ is recurrent, i.e. for any subset $A$ of positive measure and for all $z \in A$ (a.e.) $f^{n}(z) \in A$ for infinitely many positive integers $n$. (The relationship between recurrence and ergodicity of inner functions is not what one would expect, see Doering and Mané.)

Let $\Omega$ be a parabolic domain with attractive boundary point $w$ (i.e. Denjoy-Wolff point). By the classical description of the petals we see that at $w$ the domain $\Omega$ is asymptotic to a sector of angle $2 \pi / k$, where we can assume (without loss of generality, see Beardon $[2$, p. 116-122])

$$
F(z)=w+(z-w)-(z-w)^{k+1}+O\left((z-w)^{2 k+1}\right) .
$$

Supposing that the conformal mapping $h$ maps 1 to $w$ then

$$
h(z)=w+a_{1}(z-1)^{k / 2}+\ldots .
$$

Thus by a simple computation for any $\alpha>1 / 2$ for large enough $n$ :

$$
1-\left|f^{n}(0)\right|>\frac{1}{n^{\alpha}}
$$

Thus as in [6] we obtain from Lemma A the first part of:

LEMMA 2. Let $\Omega$ be a foreward invariant simply connected component of set of normality of a meromorphic function $F$ with finite Denjoy-Wolff point. Then the corresponding inner function $f$ is ergodic. Furthermore for any set $E \subset \partial \mathbb{D}$ with $f(E) \subset E$ (a.e.) we have $\lambda(E)=0$ (a.e.) or $E=\partial \mathbb{D}$ (a.e.).

To prove the last part of this lemma we define for fixed positive integer $m$

$$
G=\left\{z \in \partial \mathbb{D}-E: f^{m}(z) \in E\right\} .
$$

Now if $\lambda(E)>0$ then $f^{k}(z) \in E$, which is disjoint from $G$, for $k>m-1$ as $f$ is recurrent $\lambda(G)=0$, for all $m$. Therefore $E$ is 
backwards as well as foreward invariant. Hence as $f$ is ergodic we have the result required.

In order to prove our theorems we use conformal pullback to produce inner functions $f_{1}$ and $f_{2}$. The unit circle has subsets $E$ and $G$ both of positive length and there is a (mutually) absolutely continuous bijection $\phi: E \rightarrow G$ so that on $G$

$$
f_{2}=\phi \circ f_{1} \circ \phi^{-1}
$$

Our basic tool is the following result proved in [8],

THEOREM H1. Suppose that $f_{1}, f_{2}$ are ergodic inner (rational) functions. Now suppose there is an absolutely continuous homeomorphism (bijection) $\phi: \partial \mathbb{D} \rightarrow \partial \mathbb{D}$ so that

$$
f_{2}=\phi \circ f_{1} \circ \phi^{-1}
$$

on $\partial \mathbb{D}$ (a.e.). Then there is a Möbius transformation $T$ of the disk onto itself:

$$
\phi=T
$$

REMARK. Notice that our result is somewhat stronger for finite Blaschke products. This will be used in the proof of Theorem 4 .

3. Boundary values of conformal mappings. In this section we collect some basic ideas on the boundary behaviour of conformal maps $h: \mathbb{D} \rightarrow \Omega$. A full account may be found in Pommerenke [13].

For a point $w \in \partial \mathbb{D}$ which is the endpoint of an open Jordan arc $\alpha$ contained inside $\Omega$ there is a point $z \in \partial \mathbb{D}$ so that $z$ is the endpoint of $h^{-1}(\alpha)$ and $h$ has radial limit $w$ at $z$. We want to consider more complicated geometric behaviour. We say that $w$ is accessible in a sector if there is an open triangle in $\Omega$ with $w$ as one vertice. The set of points on $\partial \Omega$ which are sectorial limits is denoted by $S$. By a theorem of McMillan the harmonic measure $d \omega$ and length $d \lambda$ are mutually absolutely continuous on $S$. Also on $S$, except for a set of measure zero, every point has a "one sided tangent". There may also be a nontrivial set $R$ complementary to $S$, where up to a set of zero harmonic measure every point $w=h(z)$ is a twist point. This means that $\arg (h(r z)-w)$ is unbounded above and below as $r \rightarrow 1$. 
Next we need to consider disjoint simply connected domains $\Omega_{1}$ and $\Omega_{2}$ with harmonic measures $d \omega_{1}$ and $d \omega_{2}$. We make use of a basic result of Bishop, Carleson, Garnett and Jones [3] who prove

THEOREM B. The combined boundary $\partial \Omega_{1} \cup \partial \Omega_{2}$ has a partition into disjoint sets $S_{1} \cap S_{2}, B_{1}$ and $B_{2}$ where $\omega_{1}\left(B_{2}\right)=\omega_{2}\left(B_{1}\right)=0$. The set $S_{1} \cap S_{2}$ consists of all boundary points which are accessible in a sector from simultaneously both $\Omega_{1}$ and $\Omega_{2}$. Furthermore almost all (harmonic measure or $d \lambda$ it does not matter) points of $S_{1} \cap S_{2}$ are points where the two domains are mutually tangent.

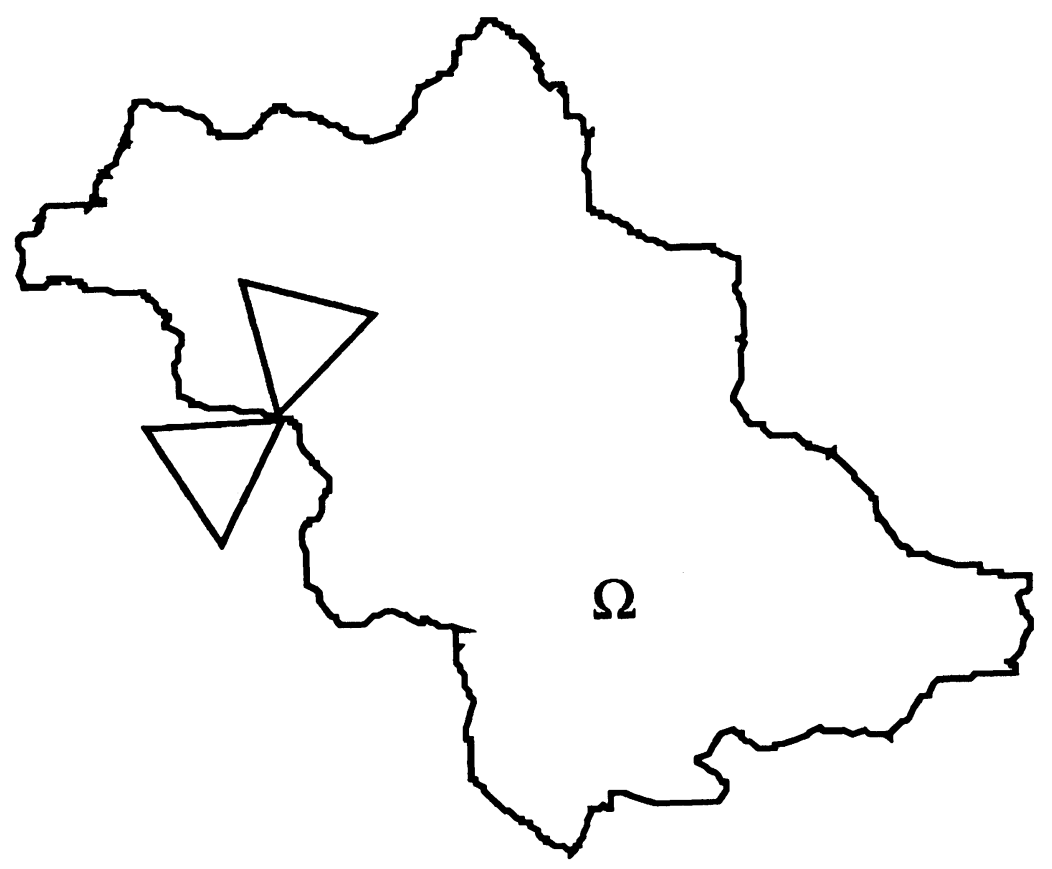

We are now ready to apply these concepts to ergodic inner functions. First we allow our domains $\Omega_{1}$ and $\Omega_{2}$ to be foreward invariant simply connected domains of the region of normality of a meromorphic function $F$. Now, except for a countable number of points, $F$ and its iterates are locally conformal. Thus the set $S_{1} \cap S_{2}$ is foreward and hence by Lemma 2 backward invariant. When we pullback to the inner functions $f_{j}$ we obtain invariant sets $E_{j}=h_{j}^{-1}\left(S_{1} \cap S_{2}\right)$. By 
the previous section our inner functions are ergodic and have nontrivial invariant sets. Thus the sets $S_{1} \cap S_{2}$ have harmonic measure 1 or 0 in both domains. Furthermore if the two harmonic measures are not singular then, by Theorem B, we have that $S_{1} \cap S_{2}$ has harmonic measure 1 in both domains. From this we construct the mapping

$$
\phi=h_{2}^{-1} \circ h_{1}
$$

defined first on a subset of positive measure on the unit disk. Thus $\phi$ may be extended to an absolutely continuous bijection defined on the unit circle (a.e). Finally by simple topology, as order is preserved, $\phi$ extends continuously to an absolutely continuous homeomorphism of the unit circle. Thus from the conjugation

$$
f_{j}=h_{j}^{-1} \circ F \circ h_{j}
$$

we have proved

LEMMA 3. If $S_{1} \cap S_{2}$ has positive measure then there is an absolutely continuous homeomorphism $\phi: \partial \mathbb{D} \rightarrow \partial \mathbb{D}$ so that $f_{2}=\phi \circ f_{1} \circ \phi^{-1}$ (a.e. on $\left.\partial \mathbb{D}\right)$.

4. Proof of Theorems 1-3. From previous results we have first to prove Theorem 3. Assuming that harmonic measure is not singular then by applying Theorem H1 to Lemma 3 we obtain a Möbius $\operatorname{map} T: \partial \mathbb{D} \rightarrow \partial \mathbb{D}$ so that

$$
h_{2} \circ T=h_{1}(\text { a.e. on } \partial \mathbb{D}) \text {. }
$$

Up till now we had some freedom in our normalization of the Riemann maps. These may now be normalised so that we can assert that $T=z$ or $T=\bar{z}$. Consider the first case. Thus the conformal maps agree (a.e.) on the unit circle. Hence they have the same Laurent coefficients for the expansions in the powers of $z$. In other words $h_{2}$ is the analytic continuation of $h_{1}$. So there is a bilinear mapping $h$ so that $h_{1}(z)=h(z)$ and $h_{2}(z)=h(z)$ for $|z|>1$. In particular the domains have mutual boundary which is a circle/line.

In the second case $T$ must be antilinear. Consequently

$$
\left.h_{2}\left(\frac{1}{z}\right)=h_{1}(z) \text { (a.e. on } \partial \mathbb{D}\right) \text {. }
$$


By comparison of Laurent coefficients again we have

$$
h_{2}\left(\frac{1}{z}\right)=h_{1}(z) \text { for all }|z| \neq 1 \text {. }
$$

This implies that $\Omega_{1}=\Omega_{2}$, contrary to our assumption. This completes the proof of Theorem 3. Theorem 2 immediately follows as by using a bilinear conjugation we can always assume any DenjoyWolff points of a rational function can be made finite. We complete the proof of Theorem 1 in the next section.

5. $\operatorname{dim}(J)>1$. Decker and Urbanski [5] define a rational map $F$ to be parabolic on $J$ if there are no critical points on $J$ but there exist at least one rationally indifferent periodic point. Now set $\delta=\operatorname{dim}(J)$. They generalise a result of Sullivan by proving that there is a unique $\delta$ - conformal probability measure $d \mu$ on $J$, i.e.

$$
\mu(F(A))=\int_{A}\left|F^{\prime}\right|^{\delta} d \mu
$$

for all $A$ in $J$ upon which $F$ is $1: 1$. They prove:

LEMMA D. If $\delta=1$ then there is a positive finite constant $c$ so that $d \mu=c d \lambda$.

Let us now consider the case that $J$ is a Jordan curve. There can be no critical points on $J$, as these would cause branching. If neither of the attractive points lie on $J$ then $F$ is expanding. So this is the Sullivan case. In the other case any attractive point on $J$ must necessarily be rationally indifferent, in fact must be a petal of order 1 or 2 . This is the parabolic case. Now by Lemma D and Theorem 2 we deduce that $\delta=1$ only in the trivial case that $J$ is a circle/line. This also completes the proof of Theorem 1 .

This argument will not hold for Jordan arcs or dendrites which in general contain critical points.

6. Multiply connected case. It is natural to ask in what sense do these results generalise to nonsimply connected domains. That is we suppose we have a rational function $F$ with two disjoint foreward invariant components $\Omega_{j}$ on the set of normality, one of which at least is multiply connected.

First we fix one such domain which we call $\Omega$. One sees that Lemma 1 and 2 generalise without any problem. For as $\partial \Omega$ has at 
least 3 points there is a conformal mapping $h$ of the unit disk $\mathbb{D}$ onto the universal covering of $\Omega$. Furthermore as $\partial \Omega$ in fact has positive logarithmic capacity a result of Nevanlinna for functions of bounded characteristic implies

$$
\lim _{r \rightarrow 1} h(r z) \in \partial \Omega \quad(z \in \partial \mathbb{D} \text { a.e. }) .
$$

Thus as before $f \equiv h^{-1} \circ F \circ h$ will be inner.

The simplest case of a nonsimply connected domain is when $\Omega_{1}$ say is a Herman ring. This is the only other finitely connected case. Then $F$ acting on $\Omega_{1}$ is conformally conjugate to an irrational rotation $f_{1}$ of an annullus $D_{1}=\{r<|z|<1\}$ :

$$
f_{1} \equiv h_{1}^{-1} \circ F \circ h_{1}
$$

where $h_{1}: D_{1} \rightarrow \Omega_{1}$ is the conformal mapping which maps the boundary component $\partial \mathbb{D}$ to the outside of the boundary component $\partial_{0} \Omega_{1}$. Note that $F$ leaves the boundary components of $\partial \Omega_{1}$ invariant so that the pullback to the unit disk $f$ cannot be ergodic. Now without loss of generality the other invariant domain is in the unbounded component of $\mathbb{C}-\Omega_{1}$.

There are several possibilities:

(i) $\Omega_{2}$ is simply connected (and contains $\infty$ ).

(ii) $\Omega_{2}$ is doubly connected.

(iii) $\Omega_{2}$ is infinitely connected.

First we assume that $\Omega_{2}$ is simply connected. Case (iii) shall be dealt with later. For case (i) we pullback $\Omega_{2}$ to $D_{2}=\{1<|z|\}$ by conformal mapping $h_{2}: D_{2} \rightarrow \Omega_{2}$ taking $\partial \mathbb{D}$ to the boundary component $\partial \Omega_{2}$. So we obtain "inner functions" $f_{j}: D_{j} \rightarrow D_{j}$ as usual.

In this section we considerably weaken the hypothesis that the harmonic measures are nonsingular. All we require is that

$$
\omega_{j}\left(\partial_{0} \Omega_{1} \cap \partial \Omega_{2}\right)>0 \text {, for } j=1 \text { and } 2 \text {. }
$$

Now as $\partial_{0} \Omega_{1} \cap \partial \Omega_{2}$ is foreward invariant under $F$ by Lemma 2

$$
\omega_{2}\left(\partial_{0} \Omega_{1} \cap \partial \Omega_{2}\right)=1
$$


Also as $f_{1}$ is an irrational rotation there are no nontrivial foreward invariant sets. Thus

$$
\omega_{1}\left(\partial_{0} \Omega_{1} \cap \partial \Omega_{2}\right)=\omega_{1}\left(\partial_{0} \Omega_{1}\right) .
$$

Thus in particular $\partial_{0} \Omega_{1}=\partial \Omega_{2}$ although this alone does not imply that there may be other components of the set of normality which lie between the two invariant domains. Under these circumstances it is easy to construct the bijection $\varphi$ giving the conjugation from $f_{1}$ to $f_{2}$ on $\partial \mathbb{D}$. First observe that for any continua $K$ in $\partial_{0} \Omega_{1}$ there is a unique subarc $I_{j} \subset \partial \mathbb{D}$ corresponding to $K$ under $h_{j}$ in the prime-end topology. Now by the above result on harmonic measure

$$
\omega_{1}(K)>0 \Longleftrightarrow \omega_{2}(K)>0 .
$$

Thus in mapping $I_{1}$ to $I_{2}$ there is no collapsing of arcs to points or vice-versa. Consequently, as by simple topology order is preserved, the bijection $\varphi$ is defined, however we do not have that it is nonsingular merely that it is a homeomorphism.

Now as $f_{1}$ is just an irrational rotation we must have that its conjugate $f_{2}$ is $1: 1$ and is therefore a rotation too. By the classification of invariant domains in this case $\Omega_{2}$ is also a Herman ring. As this was the second case we now assume it. So we deal with case (ii). In this case we pullback $\Omega_{2}$ to $D_{2}=\{1<|z|<s\}$ by conformal mapping $h_{2}: D_{2} \rightarrow \Omega_{2}$ taking $\partial \mathbb{D}$ to the inner boundary component $\partial_{i} \Omega_{2}$. So we obtain "inner functions" $f_{j}: D_{j} \rightarrow D_{j}$ which are now irrational rotations:

$$
f_{j}=e^{i \theta_{j}} z
$$

Now as above we have the dichotomy

$$
\omega_{j}\left(\partial_{0} \Omega_{1} \cap \partial \Omega_{2}\right)=0, \text { for } j=1 \text { or } 2,
$$

or

$$
\omega_{j}\left(\partial_{0} \Omega_{1} \cap \partial \Omega_{2}\right)=1, \text { for } j=1 \text { or } 2 .
$$

Once again we concentrate on the second case and produce a homeomorphism $\varphi$ of the circle onto itself so that

$$
e^{i \theta_{2}} \varphi(z)=\varphi\left(e^{i \theta_{1}} z\right), z \in \partial \mathbb{D}
$$


where $\theta_{j} / \pi$ is irrational. Let $\varphi$ have Fourier expansion

$$
\sum_{-\infty}^{+\infty} a_{k} z^{k},|z|=1
$$

Thus for each $k$ :

$$
e^{i \theta_{2}} a_{k}=e^{i k \theta_{1}} a_{k} .
$$

Therefore $a_{k}=0$ or $k \theta_{1}-\theta_{2}=2 \pi m_{k}$. But as $\theta_{2} / \pi$ is irrational there is at most one $k$ for which $a_{k} \neq 0$. But as $\varphi$ is a homeomorphism this implies that it is Möbius. Since we still have choice in the normalization of the conformal maps we may take $\varphi(z)=z$. Thus the conformal maps $h_{j}$ satisfy

$$
h_{1}(z)=h_{2}(z) \text { on } \partial \mathbb{D} \text { (a.e.). }
$$

Therefore by classical Laurent series $h_{2}$ is the analytic continuation of $h_{1}$. This implies that the common boundary $B$ of the Herman rings is the conformal image of $\partial \mathbb{D}$. Hence the "Herman Rings" are infact rings of a larger Herman Ring obtained by pasting across an analytic curve $B$. But then $B$ cannot possibly be part of the set of nonnormality! Thus in all cases the harmonic measure of the common boundary must be zero.

Finally we have to deal with the case where $\Omega=\Omega_{1}$ is infinitely connected. Now $F$ will have a (super) attractive point in $\Omega$ we see that the corresponding $f$ may be assumed to fix 0 . Then $f$ will be ergodic and recurrent. We shall now prove that either the boundary components of $\mathbb{C}-\Omega$ each have harmonic measure zero or the boundary components of of $\mathbb{C}-\Omega$ are all points (and there will be only one invariant domain). Consider any components $K$ of $\mathbb{C}-\Omega$. Suppose that the harmonic measure $\omega(K)>0$. Now let the pullback of $\partial K$ under $h$ be the set $E$. As $f$ is recurrent there is a set $E_{0} \subset E$ with $\lambda\left(E_{0}\right)>0$ and a number $n$ so that $f^{n}\left(E_{0}\right)$ is contained in $E$. But this means, as $F$ maps components of $\mathbb{C}-\Omega$ to cornponents of $\mathbb{C}-\Omega$, that $F^{n}(K)=K$. Without loss of generality $n=1$. Thus $E$ is foreward invariant under $f$. But as $f$ fixes 0 we see $\lambda\left(f^{-1}(E)\right)=\lambda(E)$ and therefore $f^{-1}(E)=E$ (a.e.). So as $f$ is ergodic $\omega(K)=1$. Thus the other components of $\mathbb{C}-\Omega$ are points. Now at least one of these components, $Q$ say, is mapped by $F$ onto 
$K$ otherwise $f$ is normal on $\mathbb{C}-K$. But then $Q$ must be a continuum too which is a contradiction. Therefore all components have zero harmonic measure. Thus we proved the multiply connected case is trivial for harmonic measures.

THEOREM 5. If $\Omega_{j}$ are disjoint foreward invariant domains at least one of which is multiply connected then $0=\omega_{1}\left(\partial \Omega_{1} \cap \partial \Omega_{2}\right)=$ $\omega_{2}\left(\partial \Omega_{1} \cap \partial \Omega_{2}\right)$.

\section{Dendrites.}

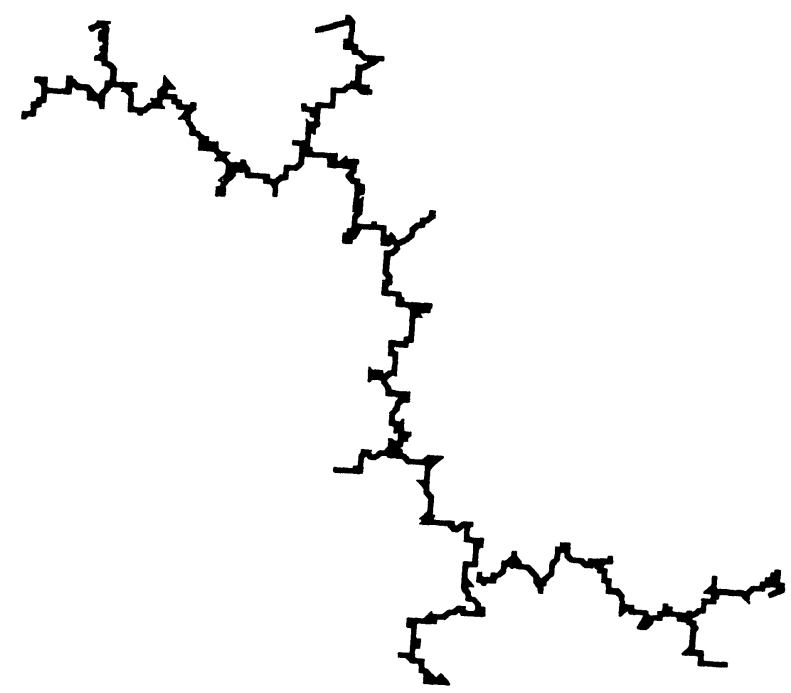

It is known, see [2, p. 257], that if $F$ is a polynomial then $J$ is the interval $[-1,1]$ if and only if $F$ is one of the Tchebyshev polynomials $T_{n}$ (or $-T_{n}$ ) where $T_{n}(\cos (t))=\cos (n t)$. In general a rational function $F$ has Julia set $[-1,1]$ if and only if

$$
F=h \circ f \circ h^{-1}, \text { where } h=\frac{1}{2}\left(z+\frac{1}{z}\right),
$$

where $f$ is an ergodic and symmetric finite Blaschke product. Now, in general but not if $F$ is a polynomial (by Hubbard's "Ecale" construction), the Teichmüller space of such rational functions is nontrivial. This means there is a quasiconformal mapping $\Phi: \mathbb{C} \rightarrow \mathbb{C}$ so that 


$$
F^{*}=\Phi \circ F \circ \Phi^{-1}
$$

is a rational function not conjugate to $F$ by any bilinear mapping. Consequently $F^{*}$ has Julia set $J^{*}=\Phi(J)$ which in general is not an interval.

Actually we shall deal with more general objects then an open Jordan arc. Assuming that $J$ and $\mathbb{C}-J$ are connected we have a single foreward invariant simply connected domain $\Omega$. In analogy to before we define $S$ to be the set of points $\zeta \in S$ so that there exists $r>0$ and directions $t_{j}, j=1$ and 2 , so that there are disjoint sectors

$\sum_{j}=\left\{z:|z-\zeta|<r, \arg \left((z-\zeta) e^{-i t_{\jmath}}\right)<\theta_{j}\right\}$, of width $2 \theta_{j}>\pi / 3$, belonging to different components of $\Omega \cap\{z:|z-\zeta|<r\}$. Now there are at most two such sectors at each point. If $J$ has (locally) finite length then almost all points of $J$ belong to $S$. Notice that $S$ is $F$ invariant (a.e.). As the conformal pullback $f$ is ergodic then either $\omega(S)=0$ or $\omega(S)=1$. In the latter case we define a bijection $\phi: \partial \mathbb{D} \rightarrow \partial \mathbb{D}$ (a.e.) as the pullback of the map swapping points in adjacent sectors - the identity, i.e. $\phi=h^{-1} \circ I \circ h$ which gives an involution (a.e.). By MacMillan's theorem $\phi$ is absolutely continuous. But as $f=h^{-1} \circ F \circ h$

$$
\begin{aligned}
\phi \circ f \circ \phi^{-1} & =h^{-1} \circ I \circ h \circ h^{-1} \circ F \circ h \circ h^{-1} \circ I \circ h \\
& =h^{-1} \circ I \circ F \circ I \circ h \\
& =h^{-1} \circ F \circ h=f,
\end{aligned}
$$

i.e. $\phi$ conjugates $f$ to itself. Consequently Theorem H1 shows that $\phi$ is Möbius. After a trivial renormalisation, if necessary, we see that we may assume

$$
\phi(z)=\bar{z}
$$

Therefore from above

$$
f(\bar{z})=\overline{f(z)} \text { and } h(\bar{z})=\overline{h(z)} .
$$

This implies that $J$ is symmetric with respect to the real axis. If $J$ is not a subinterval of the real line there will be a subset of positive 
harmonic measure where the bijection is not defined. This completes the proof of Theorem 4 .

8. Counterexample. We use quasi-conformal deformation (see Beardon [2]) to prove

THEOREM 6. There exists a meromorphic function $F$ with Julia set $J$ a Jordan curve trough $\infty$ and with two foreward invariant components $\Omega_{1}$ and $\Omega_{2}$ of $\mathbb{C}-J$ so that the harmonic measures satisfy $d \omega_{1} \sim d \omega_{2}$, indeed $d \lambda \gg d \omega_{j}$.

We begin with the functions

$$
G_{s}(z)=s z+\tan (z), s>0 .
$$

It is easy to see that for $s \geq 1$ these have Julia set $J$ equal to the real line and the upper and lower half planes are invariant. Also by [6] for $s>1$ the pullback to the disk, $f$, is not ergodic. Now in [8] we proved

ThEOREM H2. If $G_{s}: \mathbb{H} \rightarrow \mathbb{H}$ is inner but not ergodic there exists a quasiconformal mapping

$$
\Phi: \mathbb{C} \longrightarrow \mathbb{C}
$$

preserving the upper half plane so that

(i) $H_{s}=\Phi \circ G_{s} \circ \Phi^{-1}$ is meromorphic, inner on $\mathbb{H}$,

(ii) $H_{s} \neq T \circ G_{s} \circ T^{-1}$ (a.e. on $\mathbb{R}$ ) for all Möbius $T$. Also the restriction of $\Phi$ to the real axis is absolutely continuous.

Actually we do not use the last unadorned fact but the part of the construction that lies underneath it. Let $\Phi$ have complex dilatation

$$
\mu(z)=\frac{\bar{\partial} \Phi}{\partial \Phi} .
$$

By (i) the dilatation $\mu$ is $G_{s}$ invariant, i.e.

$$
\mu\left(G_{s}(z)\right)=\frac{G_{s}^{\prime}(z)}{\overline{G_{s}^{\prime}(z)}} \mu(z)
$$

and as the real line is mapped to the real line

$$
\overline{\mu(z)}=\mu(\bar{z})
$$


The important property of $\mu$ is that it is supported by a set $A$ which only has nontangential cluster points on a set $E$ on the real axis with $\lambda(E)=0$, proved in [8]. Recall that $x$ is a nontangential cluster point of $A$ if there is an angle $\theta, 0<\theta<\pi / 2$ with Stolz angle

$$
\Sigma=\{\operatorname{Im}(z)>0:|z|<r,|\operatorname{Arg}(z-x)|<\theta\} .
$$

So there is a sequence $z_{n} \in \Sigma \cap A, z_{n} \rightarrow x$.

We now define a second quasiconformal mapping

$$
\psi: \mathbb{C} \longrightarrow \mathbb{C}
$$

as a homeomorphic solution of the Beltrami equation

$$
\frac{\bar{\partial} \psi}{\partial \psi}= \begin{cases}\mu(z), & \operatorname{Im}(z)>0 \\ 0, & \text { otherwise }\end{cases}
$$

which fixes infinity, see [11].

As above $\psi$ has a $G_{s}$ invariant dilatation and therefore as in Beardon

(iii) $F_{s}=\psi \circ G_{s} \circ \psi^{-1}$ is meromorphic.

We note

(iv) $F_{s} \neq T \circ G_{s} \circ T^{-1}$ for all Möbius $T$.

Otherwise as the restriction of $\psi$ to the lower half plane is conformal we see that $\psi$ will be bilinear on the lower half plane. But then by the usual Bers theory of universal Teichmuller space the dilatation $\mu$ is trivial, and $\Phi$ will be bilinear on the real line, contradicting (ii).

The image of the real line under $\psi$ is a Jordan curve $J$ through infinity. Furthermore as $G_{s}$ has Julia set equal to the real line, $F_{s}$ has Julia set equal to $J$. Thus there are two foreward invariant components $\Omega_{1}$ and $\Omega_{2}$ of $\mathbb{C}-J$ with the harmonic measures $d \omega_{1}$, $d \omega_{2}$. By suitable choice of the conformal mappings

$$
\left\{\begin{array}{l}
h_{1}:\{\operatorname{Im}(z)>0\} \longrightarrow \Omega_{1} \\
h_{2}:\{\operatorname{Im}(z)<0\} \longrightarrow \Omega_{2}
\end{array}\right.
$$

we see that on $\mathbb{R}$

$$
\Phi=h_{2}^{-1} \circ h_{1} .
$$


Thus the harmonic measures are mutually absolutely continuous.

The second part of Theorem 6 is an application of the main theorem of [10] which with the above notation is:

THEOREM H3. Suppose that the complex dilatation of $\psi$ is supported by a set $A$ which only has nontangential cluster points on a set $E$ on the real axis with $\lambda(E)=0$ then

$$
d \lambda_{J} \circ \psi \gg d \lambda_{\mathbb{R}}
$$

This concludes the proof of Theorem 6 .

\section{REFERENCES}

[1] Aaronson, J., Ergodic theory for inner functions of the upper half-plane, Ann. Inst. H. Poincare, Sec. B, vol. XIV (1978), 233-253.

[2] Beardon, A., Iteration of rational functions, Grad. Texts in Math., no. 132, Springer-Verlag 1991.

[3] Bishop, C.J., Carleson, L., Garnett, J.B. and Jones, P.W. Harmonic measures supported on curves, Pacific J. Math., 138 (1989), 233-236.

[4] Brolin, H., Invariant sets under iteration of rational functions, Ark. Mat., 6 (1965), 103-144.

[5] Decker, M. and Urbanski, M., Geometric measures for parabolic rational maps, Ergod. Th. and Dynam. Sys., 12 (1992), 53-66.

[6] Doering, C. and Mané, R., The dynamics of inner functions, Ensaisos Matematicos, 3 (1992), 1-79.

[7] Eremenko, A.E. and Lyubich, M. Yu., The dynamics of analytic transformations, Leningrad Math. J., 1 (1990).

[8] Hamilton, D.H., Absolutely continuous conjugacies of Blaschke products, to appear in Advances in Math.

[9] , Absolutely continuous conjugacies of Blaschke products III, to appear in Journal d'Analyse.

[10] Quasiconformal mappings absolutely continuous on the circle, to appear.

[11] Letho, O and Virtanen, K.I., Quasiconformal mappings in the plane, Springer-Verlag, 1973.

[12] Nevanlinna, R., Analytic functions, Springer-Verlag 1953.

[13] Pommerenke, C., Boundary Behaviour of Conformal Maps, SpringerVerlag Grundlehren der Math. Wissen., 299 (1991).

[14] Przytycki, M., Urbanski, M., Zdunik, A., Harmonic, Gibbs and Hausdorff measures on repellers for holomorphic maps, Ann. Math., 130 (1989), $1-40$. 
[15] Shub, M. and Sullivan, D., Expanding endomorphisms of the circle revisited, Ergod. Theo. and Dynam. Sys., 5 (1985), 285-289.

[16] Sullivan, D., Conformal dynamical systems, 725-752, Lecture Notes in Math., vol. 1007, Springer-Verlag, 1983.

[17] Zdunik, A., Hausdorff measure of maximal measure for rational maps, preprint, Inst. Math. Polish Acad. Sci. Warshaw (1987).

Received September 17, 1992 and in revised form May 24, 1994. This research was supported in part by the National Science fondation.

UNIVERSITY OF MARYLAND

College PARK, MD 20742

E-mail address: dhh@math.umd.edu 



\title{
PACIFIC JOURNAL OF MATHEMATICS
}

Founded by E. F. Beckenbach (1906-1982) and F. Wolf (1904-1989)

\section{EDITORS}

Sun-Yung Alice Chang (Managing Editor)

University of California

Los Angeles, CA 90095-1555

pacific@math.ucla.edu

F. Michael Christ

University of California

Los Angeles, CA 90095-1555

christ@math.ucla.edu

Thomas Enright

University of California

San Diego, La Jolla, CA 92093

tenright@ucsd.edu

Nicholas Ercolani

University of Arizona

Tucson, AZ 85721

ercolani@math.arizona.edu
Robert Finn

Stanford University

Stanford, CA 94305

finn@gauss.stanford.edu

Vaughan F. R. Jones

University of California

Berkeley, CA 94720

vfr@math.berkeley.edu

Steven Kerckhoff

Stanford University

Stanford, CA 94305

spk@gauss.stanford.edu
Martin Scharlemann

University of California

Santa Barbara, CA 93106

mgscharl@math.ucsb.edu

\section{Gang Tian}

Courant Institute

New York University

New York, NY 10012-1100

tiang@taotao.cims.nyu.edu

V. S. Varadarajan

University of California

Los Angeles, CA 90095-1555

vsv@math.ucla.edu

\section{SUPPORTING INSTITUTIONS}

\section{CALIFORNIA INSTITUTE OF TECHNOLOGY \\ NEW MEXICO STATE UNIVERSITY \\ OREGON STATE UNIVERSITY \\ STANFORD UNIVERSITY \\ UNIVERSITY OF ARIZONA \\ UNIVERSITY OF BRITISH COLUMBIA \\ UNIVERSITY OF CALIFORNIA UNIVERSITY OF HAWAII}

\author{
UNIVERSITY OF MONTANA \\ UNIVERSITY OF NEVADA, RENO \\ UNIVERSITY OF OREGON \\ UNIVERSITY OF SOUTHERN CALIFORNIA \\ UNIVERSITY OF UTAH \\ UNIVERSITY OF WASHINGTON \\ WASHINGTON STATE UNIVERSITY
}

The supporting Institutions listed above contribute to the cost of publication of this Journal, but they are not owners or publishers and have no responsibility for its contents or policies.

Manuscripts must be prepared in accordance with the instructions provided on the inside back cover.

The Pacific Journal of Mathematics (ISSN 0030-8730) is published monthly except for July and August. Regular subscription rate: $\$ 215.00$ a year (10 issues). Special rate: $\$ 108.00$ a year to individual members of supporting institutions.

Subscriptions, orders for back issues published within the last three years, and changes of subscribers address should be sent to Pacific Journal of Mathematics, P.O. Box 4163, Berkeley, CA 94704-0163, U.S.A. Prior back issues are obtainable from Kraus Periodicals Co., Route 100, Millwood, NY 10546.

The Pacific Journal of Mathematics at the University of California, c/o Department of Mathematics, 981 Evans Hall, Berkeley, CA 94720 (ISSN 0030-8730) is published monthly except for July and August. Second-class postage paid at Berkeley, CA 94704, and additional mailing offices. POSTMASTER: send address changes to Pacific Journal of Mathematics, P.O. Box 6143, Berkeley, CA 94704-0163.

\author{
PUBLISHED BY PACIFIC JOURNAL OF MATHEMATICS at University of California, \\ Berkeley, CA 94720, A NON-PROFIT CORPORATION \\ This publication was typeset using AMS-LATEX, \\ the American Mathematical Society's TEX macro system. \\ Copyright (C) 1995 by Pacific Journal of Mathematics
}




\section{PACIFIC JOURNAL OF MATHEMATICS}

Volume 169 No. $1 \quad$ May 1995

Minimal sets of periods for torus maps via Nielsen numbers

Lluís AlsedÀ, StewART BALdWin, JAUME Llibre,

RICHARD SWANSON and WIESLAW SZLENK

Diagonalizing Hilbert cusp forms

Timothy ATWILL

A splitting criterion for rank 2 vector bundles on $\mathbf{P}^{n}$

EDOARDO BALLICO

Controlling Tietze-Urysohn extensions

MARC FRANTZ

Length of Julia curves

DAVID H. HAMILTON

On the uniqueness of capillary surfaces over an infinite strip

JENN-FANG HWANG

Volume estimates for log-concave densities with application to iterated 107 convolutions

MARIUS JUNGE

A reflection principle in complex space for a class of hypersurfaces and mappings

FRANCINE ANTOINETTE MEYLAN

Jean Bourgain's analytic partition of unity via holomorphic martingales 161

PAUL F.X. MÜLLER

Characters of Brauer's centralizer algebras 